Cahiers $d u$ MONDE RUSSE

\section{Cahiers du monde russe}

Russie - Empire russe - Union soviétique et États indépendants

$46 / 1-2 \mid 2005$

La Russie vers 1550

\title{
Les princes de Moscou face à la mort
}

Modèle monastique et sainteté lignagère (1263-1598)

\section{Pierre GONNEAU}

\section{(2) OpenEdition}

1 Journals

Édition électronique

URL : https://journals.openedition.org/monderusse/8794

DOI : $10.4000 /$ monderusse. 8794

ISSN : $1777-5388$

Éditeur

Éditions de l'EHESS

\section{Édition imprimée}

Date de publication : 1 janvier 2005

Pagination : 193-210

ISBN : 2-7132-2055-6

ISSN : $1252-6576$

\section{Référence électronique}

Pierre GONNEAU, «Les princes de Moscou face à la mort », Cahiers du monde russe [En ligne], 46/1-2 | 2005, mis en ligne le 01 janvier 2007, consulté le 04 septembre 2022. URL : http://

journals.openedition.org/monderusse/8794; DOI : https://doi.org/10.4000/monderusse.8794 
chercher : repérer : avancer

Cet article est disponible en ligne à l'adresse :

http://www.cairn.info/article.php?ID REVUE=CMR\&ID NUMPUBLIE=CMR 461\&ID ARTICLE=CMR 4610193

Les princes de Moscou face à la mort. Modèle monastique et sainteté lignagère (1263-1598)

par Pierre GONNEAU

\section{Editions de l'EHESS | Cahiers du monde russe}

2005/1-2 - Vol 46

ISSN 1252-6576 | ISBN 2713220556 | pages 193 à 210

Pour citer cet article :

—GONNEAU P., Les princes de Moscou face à la mort. Modèle monastique et sainteté lignagère (1263-1598), Cahiers du monde russe 2005/ 1-2, Vol 46, p. 193-210.

Distribution électronique Cairn pour les Editions de l'EHESS.

(C) Editions de l'EHESS. Tous droits réservés pour tous pays.

La reproduction ou représentation de cet article, notamment par photocopie, n'est autorisée que dans les limites des conditions générales d'utilisation du site ou, le cas échéant, des conditions générales de la licence souscrite par votre établissement. Toute autre reproduction ou représentation, en tout ou partie, sous quelque forme et de quelque manière que ce soit, est interdite sauf accord préalable et écrit de l'éditeur, en dehors des cas prévus par la législation en vigueur en France. Il est précisé que son stockage dans une base de données est également interdit. 


\title{
LES PRINCES DE MOSCOU FACE À LA MORT
}

\author{
Modèle monastique et sainteté lignagère \\ (1263-1598)
}

\author{
« Царьский убо сань дръжаше, и аггелскы живяше » \\ Слово о житьи и о преставлении великаго князя \\ Дмитрия Ивановича, царя рускаго 1 \\ « Alors qu'il avait rang de roi, c'est en ange [i.e. en moine] qu'il vivait » \\ Dit sur la vie et le trépas du grand-prince Dmitrij Ivanovič, tsar russe
}

Cette étude cherche à compléter et à nuancer les résultats d'une première ébauche qui portait seulement sur la période allant de la mort de Dmitrij Donskoj (1389) à celle du dernier frère de Vasilij III, Andrej Ivanovič (1537)2. Nous voudrions donner une vision d'ensemble du comportement des fils de la maison de Moscou au moment du trépas. Nous tenterons d'établir, chaque fois que cela est possible, l'état - laïque ou monastique - dans lequel le prince est mort, ses dispositions testamentaires, son lieu de sépulture et sa réputation posthume.

Notre enquête embrasse les dix générations de la lignée, depuis son fondateur, Daniil († 1303), jusqu'à son dernier descendant, le tsar Fedor, fils d'Ivan le Terrible $(†$ 1598). On dénombre ainsi 84 individus, auxquels nous avons ajouté le grandpère et le père de Daniil de Moscou, Jaroslav Vsevolodovič († 1246) et Aleksandr Jaroslavič Nevskij (†1263), car leur souvenir a contribué à former les comportements moscovites. Sur cette liste de 86 noms, 18 sont des enfants morts en bas âge, incapables de prendre des dispositions personnelles, qui ne figurent que pour mémoire. On compte également 19 princes morts en exil, en prison, ou sur

1. Pamjatniki literatury Drevnej Rusi [ci-après $P L D R$ ], t. 4 : XIV-seredina $X V$ veka, M., 1981, p. 214.

2. P. Gonneau, « L'ideale della santità monastica e la dinastia moscovita (1389-1537) : il principe di fronte alla morte », in Forme della santità russa : atti dell'VIII Convegno ecumenico internazionale di spiritualità ortodossa, sezione russa, Bose, 21-23 settembre 2000, Magnano, 2002, p. 96-108. 
l'échafaud. Ceux-là ne doivent pas être négligés : quoique dépouillés de la plupart de leurs prérogatives, ils reprennent parfois l'initiative au moment de mourir ; en outre, les circonstances de leur trépas déterminent s'ils sont agrégés ou non aux autres défunts de la lignée.

Nos sources sont avant tout narratives. Le matériau principal peut être trouvé dans les chroniques rédigées à Vladimir-sur-la-Kljaz'ma puis à Moscou, par les clercs de la chaire métropolitaine, ou dans l'entourage des grands-princes aux XIVe, $\mathrm{XV}^{\mathrm{e}}$ et $\mathrm{XVI}^{\mathrm{e}}$ siècles. Il arrive cependant que des compilations (svody) émanant d'autres centres permettent de préciser ou de rectifier certains faits, ou une date ${ }^{3}$. Les chroniques donnent en général de courtes notices nécrologiques, mais elles ont aussi conservé quelques récits plus amples, sur une mort particulièrement édifiante. Le plus important est le Récit sur le trépas de Vasilij III sur lequel nous avons déjà eu l'occasion de travailler ${ }^{4}$.

Les textes hagiographiques constituent un deuxième groupe de sources, même s'ils sont parfois incorporés, eux aussi, dans les compilations de chroniques. Quatre rejetons mâles de la dynastie moscovite ont fait l'objet d'une vénération plus ou moins aboutie. Aux deux plus fameux, Aleksandr Nevskij et Dmitrij Donskoj, ont été consacrées des hagio-biographies qui célèbrent autant leurs prouesses militaires que leur piété. La Vie d'Ivan-Ignace de Vologda commémore un prince qui ne régna pas, mais passa son existence dans les souffrances, en prison ; après sa mort, il devint un saint local des pays d'Ustjug et de Vologda ${ }^{5}$. Enfin, le tsarévitch Dmitrij, mort dans des circonstances suspectes en 1591 à Uglič, a été vénéré comme un martyr, mais les textes et l'iconographie le concernant datent pour l'essentiel de l'époque des Romanov que nous ne traiterons pas ici6'.

Les sources diplomatiques sont plus rares et souvent laconiques. Nous possédons les testaments de dix-huit membres de la dynastie moscovite, depuis Ivan Ier jusqu'à Ivan le Terrible, mais ils enregistrent essentiellement des partages successoraux et les dispositions religieuses y sont assez rares ${ }^{7}$. Les chartes conservées par plusieurs monastères, leurs livres de dons (vkladnye knigi) ou leurs pitanciers

3. Ibid., p. 88-92. Pour une présentation plus détaillée de ces sources, voir V. Vodoff, "L'historiographie dans la Russie ancienne », in Histoire de la littérature russe, t. 1 : Des origines aux Lumières, Paris, 1992, p. 194-207 ; Ja. S. Lur'e, Dve istorii Rusi XV veka : rannie i pozdnie, nezavisimye i oficial'nye letopisi ob obrazovanii Moskovskogo gosudarstva, Paris $\mathrm{SPb} ., 1994$ (Collection historique de l'Institut d'études slaves, 35).

4. PLDR, t. 7 : Seredina XVI veka, M., 1985, p. 18-47 et P. Gonneau, « Vasilij III et Charles Quint face à la mort : ou le prince et l'habit des anges (essai d'étude comparative) », in Être catholique - être orthodoxe - être protestant : confessions et identités culturelles en Europe médiévale et moderne, Wroclaw, 2003, p. 235-251.

5. PLDR, t. 3 : XIII vek, M., 1981, p. $426-439$ (Vie d'Aleksandr), et PLDR, t. 4, p. 208-229 (Vie de Dmitrij) ; Jaroslavskie eparhal'nye vedomosti, 28, 1873, č. Neofficial'naja, p. 223-228 (Vie d'Ivan-Ignace).

6. S. F. Platonov, Drevnerusskie skazanija i povesti o Smutnom vremeni XVII veka, kak istoričeskij istočnik, 2e éd., SPb., 1913, p. 363-368.

7. L. V. Čerepnin, éd., Duhovnye i dogovornye gramoty velikih i udel'nyh knjazej XIV-XVI $V V$., M.-L., 1950 [ci-après $D D G$ ] ; traduction anglaise, R. C. Howes, The Testaments of the Grand Princes of Moscow, Ithaca (NY), 1967. 
(kormovye knigi) sont souvent plus riches de renseignements. Malheureusement, une partie de cette documentation demeure inédite et plusieurs livres ont été compilés tardivement, nous privant sans doute des informations les plus anciennes. Dans le cas de la Trinité Saint-Serge qui fut, dès l'époque de Dmitrij Donskoj, très proche de la dynastie moscovite, le Pitancier date de 1592, le premier Livre des dons a été rédigé en 1638/1639 et le deuxième en 1673, à la suite de quoi il fut tenu à jour jusque dans les années 30 du XVIII siècle ; les chartes et les cartulaires du monastère n'ont été publiés que jusqu'à l'année $1527^{8}$.

Du dépouillement de cette documentation se dégagent d'emblée deux types bien distincts. Le premier est celui du prince-moine qui décide, pour assurer son salut, de prendre l'habit religieux sur son lit de mort. Le second, au contraire, semble avoir des relations limitées, on serait tenté de dire protocolaires, ou en tout cas intermittentes, avec l'ordre monastique. Mais un examen plus approfondi permet de définir un troisième type, intermédiaire, celui du « prince-nourricier » qui n'entre pas dans les ordres, mais suit la direction spirituelle d'un moine, ou manifeste une dévotion et une générosité particulières envers ses abbayes favorites. Nous présenterons successivement ces trois modèles.

\section{Le prince-moine : la prise d'habit in articulo mortis}

Sur dix générations et pour un total de 84 individus, on dénombre 10 moines dans la famille moscovite. Avec Aleksandr Nevskij, le total est porté à 11 moines en onze générations. Mais les vocations ne sont pas également réparties dans le temps et surtout elles ne concernent pas le même type d'homme selon les périodes.

Pendant un siècle, de 1263 à 1359, on peut parler à juste titre d'un «modèle monastique », inauguré par l'exemple édifiant d'Aleksandr Nevskij. Epuisé par un ultime séjour à la Horde, où il n'a pas épargné sa peine pour alléger la misère de ses sujets opprimés par le khan, Aleksandr est incapable d'atteindre sa capitale, Vladimir, mais revêt la bure avant d'expirer' ${ }^{9}$. On remarque qu'il est le seul de ses sept frères à réclamer la tonsure ${ }^{10}$. En revanche, en l'espace de quatre générations, d'Aleksandr Nevskij à Ivan II le Bel, on compte cinq moines. Quatre d'entre eux ont été prince de Moscou et/ou grand-prince de Vladimir et ce sont les quatre ascendants directs de Dmitrij Ivanovič Donskoj. S’y ajoute son grand-oncle, Afanasij

8. «Kormovaja kniga XVI veka biblioteki Troickoj Sergievoj Lavry n 8211.92 » in A. V. Gorskij, Istoričeskoe opisanie sv. Troice-Sergievy Lavry, II, M., 1892, p. 35-56; E. N. Klitina, T. N. Manušina, T. V. Nikolaeva, éds., Vkladnaja kniga Troice-Sergieva monastyrja, M., 1987 ; S. B. Veselovskij, I. A. Golubcov, éds., Akty social'no-ekonomičeskoj istorii severo-vostočnoj Rusi konca XIV-načala XVI v., t. 1 et 3, M., 1952-1964 [ci-après ASEI] ; Akty russkogo gosudarstva 1505-1526 gg., M., 1975.

9. PLDR, t. 3, p. 436-438.

10. Voir les mentions du trépas des frères d'Aleksandr dans le Moskovskij letopisnyj svod konca XV veka, M., 1949, p. 125, 141, 142, 145, 150, 151 (Polnoe sobranie russkih letopisej, 25) et dans le Rogožskij letopisec, Petrograd, 1922, col. 31-33 (Polnoe sobranie russkih letopisej, 15.1) [ci-après : PSRL]. 
Danilovič (voir tableau, $n^{\circ} 2.4$ ), qui avait souvent représenté à Novgorod les intérêts du souverain moscovite ${ }^{11}$. Il s'agit donc de personnages éminents qui couronnent une existence temporelle bien remplie par l'entrée dans les ordres. Quant à Jurij Danilovič (2.1), Semen le Fier (3.1) et Andrej Ivanovič (3.4) qui demeurent dans le monde jusqu'au bout, on sait que le premier fut assassiné à la Horde et les deux autres moururent brutalement de la peste.

On observe ensuite une rupture, puisqu'aucun représentant de la génération de Dmitrij Donskoj ne prend l'habit. Cela s'explique facilement pour les six fils de Semen le Fier qui moururent tous en bas âge, mais il paraît très étonnant que ni Dmitrij Ivanovič, ni son cousin Vladimir Andreevič, qui ont pourtant régné longuement sur leurs territoires respectifs, n'aient pris l'habit au moment de mourir.

Les $5^{\mathrm{e}}, 6^{\mathrm{e}}$ et $7^{\mathrm{e}}$ générations de la dynastie moscovite confirment ce revirement : on y dénombre seulement trois moines sur 31 princes et il s'agit de personnages effacés : Ivan Dmitrievič $(5.5)^{12}$, Semen Vladimirovič $(5.10)^{13}$ et Ivan Jur evič $(6.6)^{14}$. Mais l'information la plus significative que nous recueillons pour tout le $\mathrm{XV}^{\mathrm{e}}$ siècle concerne Vasilij II († 1462). Ce souverain qu'une piété personnelle liait fortement à l'abbaye de la Trinité Saint-Serge et qui avait envisagé de s'y retirer dès 1446, lorsqu'il fut renversé par son cousin Dmitrij Šemjaka, exprima le désir d'entrer dans les ordres durant le carême 1462, mais « on ne le laissa pas faire ${ }^{15}$. Non seulement la prise d'habit in extremis n'apparaît plus comme la meilleure issue, mais elle est désormais combattue. On devine que les raisons de l'opposition à cette décision sont politiques. Vasilij II avait associé au trône, dès 1448, son fils aîné, le futur Ivan III, pourtant la cour craignait le désordre que pourrait provoquer la cohabitation entre un ancien souverain devenu moine et son successeur.

Après la mort de Vasilij II, le jeu politique moscovite, loin de se détendre, est de plus en plus marqué par la méfiance et les rivalités familiales. Si aucun cadet ne parvient à renverser le souverain régnant, celui-ci ne se prive pas d'interner ou de pousser à l'exil plusieurs de ses parents. On devine que la sérénité nécessaire à la prise d'habit fait défaut. De fait, les deux exemples d'entrée dans les ordres de la huitième génération sont particulièrement dramatiques. En 1522 ou 1523, c'est un jeune prince emprisonné, Ivan Andreevič (8.7), qui couronne près de trente ans de réclusion forcée en demandant à être admis dans les ordres ; il meurt en odeur de sainteté à cause de

11. PSRL, t. 25, p. 160,166-167 ; Novgorodskaja pervaja letopis’'staršego i mladšego izvodov, M.-L., 1950,p. 94-96, 335-339, 457.

12. PSRL, t. 25, p. 221.

13. Selon les sources, il s'agit de Semen ou de son frère Andrej, mais la première identification nous semble plus probable, voir P. Gonneau, «L'Ideale della santità monasticà... », art. cit., p. 106-107.

14. PSRL, t. 24, p. 182 et G.V. Semenčenko, «Neizvestnyj syn Jurija Galickogo i političeskaja bor'ba na Rusi v načale 30-h gg. XV veka », Vspomogatel'nye istoričeskie discipliny, 22, 1991,p. 188-193.

15. PSRL, t. 18 , p. 215 ; t. 25 , p. 278 ; t. 12 , p. $114-115$; sur les liens de Vasilij II avec la Trinité, voir. P. Gonneau, La Maison de la Sainte Trinité : un grand monastère russe du Moyenâge tardif (1345-1533), Paris, 1993, p. 160-182 et DDG, n 61, p. 196. 
son exemplaire résignation dans l'épreuve ${ }^{16}$. En décembre 1533, le grand-prince de Moscou en personne, Vasilij III, doit livrer une véritable bataille sur son lit de mort pour être autorisé à revêtir l'habit des anges ${ }^{17}$. Si le clergé, emmené par le métropolite Daniel, appuie sa décision, certains de ses conseillers et son propre frère, Andrej Ivanovič (8.6), tentent à plusieurs reprises de le dissuader. Dans cette joute, il n'est pas question des ascendants qui ont déjà reçu la tonsure. Au contraire, l'argument lignager est utilisé par Andrej pour s'opposer à la vocation monastique :

Vladimir, le grand-prince de Kiev, est mort sans se faire moine, en a-t-il été jugé indigne du repos du juste? D'autres grands-princes sont morts sans se faire moines et n'ont-ils pas trouvé le repos en compagnie des justes ? ${ }^{18}$.

Il est clair que Vasilij III ne partageait pas cette conviction, puisqu'il prit l'habit, d'une part, et fit en outre inscrire dans l'obituaire (sinodik) du monastère de la Dormition Saint-Joseph de Volokolamsk tous les princes de la dynastie de Moscou, ainsi que leurs ancêtres de la période kiévienne, à l'exclusion toutefois de Vladimir qui, lui seul, ne devait pas avoir besoin des prières de la confrérie ${ }^{19}$. Andrej Ivanovič intervenait parce qu'il craignait les troubles que pouvait causer la retraite de Vasilij III, alors que son héritier, le futur Ivan le Terrible, n'avait que trois ans. De fait, une semaine après la mort du souverain, son frère aîné, Jurij, fut arrêté. Le tour d'Andrej vint trois ans plus tard et les deux oncles d'Ivan le Terrible finirent leurs jours en prison ${ }^{20} \ldots$

Le cas d'Ivan le Terrible est, quant à lui, extrêmement délicat à interpréter. On sait qu'il fit à plusieurs reprises mine d'abandonner le pouvoir et l'expérience de l'opričnina tout entière (1565-1572) pourrait être lue comme la parodie démente de la fondation d'un ordre monastique ${ }^{21}$. Les opričniki, qui composaient alors l'entourage d'Ivan, n'étaient-ils pas une troupe d'hommes choisis, revêtus d'un uniforme particulier, liés par un vœu d'obéissance à la personne du souverain/abbé, tout dévoués à la règle nouvelle qu'il venait d'instaurer sur un territoire réservé d'où devaient être exclus les serviteurs jugés indignes ? En 1572, le tsar rédigea un très prolixe testament qui tranche sur ceux de ses prédécesseurs en ce qu'il comporte un long enseignement à ses fils Ivan et Fedor où l'on retrouve des échos du Récit sur le trépas de Vasilij III,

16. Ivan Ignace meurt le 19 mai 1523 (7031) d'après une source novgorodienne (PSRL, t. 3 , p. 198) ou 1525 (7033) d'après sa Vie (Jaroslavskie eparhal'nye vedomosti, 28, 1873, č. Neofficia'naja, p. 224); il avait été arrêté, en compagnie de son père, Andrej l'Aîné, et de son frère, Dmitrij, le 20 septembre 1491, cf. PSRL, t. 18, p. 275.

17. Voir P. Gonneau, « L'Ideale della santità monastica... », art. cit., p. 97-102 et , « Vasilij III et Charles Quint face à la mort », art. cit., p. 240-242, 248.

18. PLDR, t. 7, Seredina XVI veka, M., 1985, p. 40.

19. S. V.Sazonov, «K probleme vosprijatii smerti v srednevekovoj Rusi », in Russkaja istorija : problemy mentaliteta, M., 1994, p. 52.

20. PSRL, t. 13, p. 115, 121.

21. Sur cette période, voir S. B. Veselovskij, Issledovanija po istorii opričniny, M., 1963 ; A. A. Zimin, Opričnina Ivana Groznogo, M., 1964 ; R. G. Skrynnikov, Opričnyj terror, L., 1969 ; Id., Rossija posle opričniny : očerki političeskoj i social'noj istorii, L., 1975. 
mais aussi de la fameuse Instruction (Poučenie) du prince de Kiev Vladimir Monomaque $^{22}$. L'année suivante, Ivan le Terrible écrivit aux frères de Saint-Cyrille de Beloozero une longue épître, digne des admonestations du fondateur de ce prestigieux monastère, où il exprimait le vœu de rejoindre un jour leur communauté23.

Toutefois, jusqu'au bout, Ivan le Terrible conserva la réalité du pouvoir et il est la figure impériale par excellence, présidant le fameux synode des Cent chapitres (Stoglav, 1551) qui prescrit d'interdire toute nouvelle acquisition de terre par l'Église. Il est vrai que cette interdiction dut être renouvelée plusieurs fois, en particulier en 1580/1581, ce qui prouve son inefficacité. L'Anglais Jerome Horsey affirme qu'à cette époque Ivan le Terrible manifesta une assez forte hostilité aux moines. Il ajoute que, voulant contraindre le clergé régulier à renoncer à ses richesses, le Terrible alla jusqu'à livrer des frères à des ours féroces. L'épisode semble être légendaire : il importait de montrer à la fois la cruauté du tsar et l'excessive richesse du clergé régulier qu'Ivan aurait voulu confisquer, à l'exemple du « valorous King Henry the eighth of England ${ }^{24}$. Le parti-pris antimonastique de Horsey l'empêche probablement de décrire fidèlement l'agonie d'Ivan en disant ce qui pour nous est l'essentiel : il reçut la tonsure. Le compte rendu anglais est sans doute exact pour ce qui est des conditions matérielles : le 18 mars 1584, au sortir d'un bain, Ivan veut se distraire en compagnie de favoris, dont Boris Godunov, quand il est pris d'un malaise :

Great outcry and stir; one sent for aqua-vitae, another to the apothecary for marigold and rosewater and to call his ghostly father and the physicians. In the mean he was strangled and stark dead ${ }^{25}$.

Les sources russes rapportent que son confesseur, Feodosij Vjatka, le revêtit d'un habit monastique et lui donna en religion le nom de Jonas. La chronique dite du monastère Saint-Alexandre Nevskij, confirme formellement qu'Ivan IV avait « pris l'habit des anges ${ }^{26}$. Peu avant sa mort, le tsar avait adressé aux monastères du pays une charte dans laquelle il demandait aux frères de lui accorder leur pardon et de prier pour la rémission de ses péchés ${ }^{27}$.

22. $D D G, \mathrm{n}^{\circ} 104$, p. 426-444, à comparer avec $P L D R$, t. 7, p. 18-47 et $P L D R$, t. 1, p. 393-413.

23. D. S. Lihačev, Ja. S. Lur'e, éds., Poslanija Ivana Groznogo, M., 1951, p. 162-192, en particulier p. 164 : «аз грешный вам известих желание свое о пострижении... И мне мнится окаянному, яко исполу есмь чернец : аще и не отложих всякаго мирскаго мятежа, но уже рукоположение благословления ангельскаго образа на себе ношу ». Voir aussi M. Ferrand, « Ivan le Terrible écrivain : l'Épître aux moines du Lac Blanc (1573) », Slovo, 17, 1995-1996, p. 99-139 [Mélanges François de Labriolle], en particulier p. 101.

24. L. E. Berry, R. O. Crummey, éds., Rude and Barbarous Kingdom : Russia in the Accounts of Sixteenth-Century English Voyagers, Madison - Milwaukee - Londres, 1968, p. 280285 ; Horsey aurait pu s'inspirer de l'histoire de l'exécution de l'archevêque de Novgorod, Léonid, en 1575, qui aurait été recouvert d'une peau d'ours et livré à une meute de chiens.

25. Ibid., p. 306.

26. S. M. Solov'ev, Istorija Rossii s drevnejših vremen : toma 5-6, M., 1989, p. 681 (Sočinenija v vosemnadcati knigah, 3) ; B. N. Florja, Ivan Groznyj, M., 2002, p. 387-388 (Žizn' zamečatel'nyh ljudej, 1037); V. I. Koreckij, « Smert' Groznogo carja », Voprosy istorii, 9, 1979, p. 93-103 ; PSRL, t. 29, p. 219, 222 ; t. 14, p. 2, 34-35 ; t. 34, p. 229.

27. Voir Dopolnenija k aktam istoričeskim, t. 1, SPb., 1846, n 129, p. 185 [ci-après DAI]. 
La mort sous la bure reste une garantie de salut, surtout pour le premier empereur russe, conscient de son iniquité autant que de sa grandeur. Toutefois, on doit noter qu'à aucun moment la prise d'habit n'empêche le prince de reposer dans la nécropole familiale de l'archange Saint-Michel. C'est Ivan Kalita qui la fait construire et il y est inhumé alors qu'il a reçu la tonsure ${ }^{28}$. Après lui, la grande majorité des mâles de la dynastie moscovite reposera là, quel que soit l'état dans lequel ils sont morts.

\section{Le trépas sans intercession monastique?}

Le modèle monastique a donc connu un regain de faveur à la cour de Moscou avec Vasilij III et son fils Ivan le Terrible. Mais ces deux exceptions spectaculaires ne font peut-être que confirmer la règle tacitement instaurée sous le règne de Dmitrij Donskoj : le souverain ne doit ou ne peut abdiquer son autorité. Dmitrij Donskoj avait eu, pourtant, des relations suivies avec Serge de Radonež, le réformateur du monachisme russe, qui fut d'ailleurs témoin de son testament et assista à ses funérailles ${ }^{29}$. Mais il est aussi avéré que Dmitrij se brouilla avec les milieux monastiques à la suite de la mort du métropolite Alexis (1378) à la place duquel il souhaitait voir un de ses favoris auquel les grands abbés de l'époque étaient hostiles ${ }^{30}$. Après la mort de Dmitrij, Vasilij Irer, Vasilij II, Ivan III et son successeur désigné Ivan Ivanovič meurent tous sans entrer dans les ordres. Nous avons vu que Vasilij II aurait voulu prendre l'habit ; en revanche, rien de tel n'apparaît chez les autres grands-princes que nous venons de citer. Les testaments que nous avons conservés et les récits sur leur trépas qui nous sont parvenus frappent au contraire par le peu de place qu'ils laissent à la dévotion ${ }^{31}$. Certes, Vasilij Irer et Vasilij II accordèrent d'importants privilèges aux monastères russes, en particulier à la Trinité Saint-Serge, mais ils agissaient d'abord pour promouvoir le relèvement de terroirs que la peste et les raids tatars avaient dévastés. Ivan III, de son côté, n'eut de cesse de restreindre les exemptions et les immunités des abbayes russes $^{32}$. À partir du règne de Vasilij III, les pèlerinages de cours, entre autres à la Trinité Saint-Serge, deviennent une des manifestations rituelles de ce que l'on pourrait appeler «l'année du souverain » qui se calque sur l'année liturgique russe. À l'occasion de ces visites, le grand-prince ou le tsar régale traditionnellement la confrérie. Les plus grandes libéralités semblent toutefois réservées aux époques où le souverain et son épouse sollicitent la naissance d'un héritier et non au temps de la mort et du deuil ${ }^{33}$.

28. PSRL, t. 25, p. 172.

29. PLDR, t. 4, p. $220 ; P S R L$, t. 25 , p. 218 et $D D G, \mathrm{n}^{\circ} 12$, p. 36.

30. G. M. Prohorov, Povest'o Mitjae : Rus'i Vizantija v epohu Kulikovskoj bitvy, L., 1978 ; J. Meyendorff, Byzantium and the Rise of Russia : A Study of Byzantino-Russian Relations in the Fourteenth Century, Cambridge, 1981, p. 199-232.

31. P. Gonneau, « L'ideale della santità monastica... », art. cit., p. 120-121.

32. P. Gonneau, La Maison de la Sainte Trinité..., op. cit., p. 157-211.

33. D. B. Miller, «The Cult of Saint Sergius of Radonezh and its Political Uses », Slavic Review, 52, 1993, p. 680-699 ; N. Shields Kollmann, « Pilgrimage, Procession and Symbolic Space in XVIth-Century Russian Politics », California Slavic Studies, 19, 1994, p. 163-181. 
Un autre groupe de princes est, logiquement, écarté des vœux monastiques. Les princes emprisonnés, à l'exception d'Ivan-Ignace de Vologda, ne demandent pas à prendre l'habit avant de mourir, ou n'y sont pas autorisés. Cela peut provenir de la simple méfiance: rien ne garantissait que le nouveau frère ne romprait pas ses vœux pour tenter de récupérer ses droits. Dans le passé lointain, en 1147, un prince de la lignée de Černigov, Igor' Ol'govič, avait été massacré par les Kiéviens qui le soupçonnaient de vouloir quitter son couvent ${ }^{34}$. En 1205, Rjurik Rostislavič, qui avait été tonsuré de force, remonte sur le trône de Kiev en se défroquant ${ }^{35}$. À une époque beaucoup plus proche, en 1447, Vasilij II fit lever par l'abbé de SaintCyrille de Beloozero sa promesse de renoncer au trône de Moscou au profit de Dmitrij Šemjaka ${ }^{36}$. Mais il y a sans doute une raison plus profondément religieuse au refus de l'entrée dans les ordres pour les princes captifs : ils n'ont pas besoin de l'habit, parce qu'ils gagnent leur salut en acceptant le martyre lent de l'emprisonnement. En effet, on remarque que les chroniqueurs ont tendance à leur conférer, au moins de façon rhétorique, les attributs de la sainteté souffrante. Ils qualifient leur trépas parfois de нужая смерть (i.e. mort dans la détresse, dans la souffrance, dans la contrainte : on serait tenté de dire « malemort » $)^{37}$, mais on trouve aussi l'expression страдальческая смерть (passion) ${ }^{38}$ qui est employée par ailleurs pour les saints princes-martyrs Boris et Gleb ou pour les princes exécutés par les Tatars à la Horde. Après cette mort sanctifiante, ils rentrent en quelque sorte dans leurs prérogatives princières, puisqu'ils sont en général enterrés avec les souverains, dans la nécropole familiale de l'archange Saint-Michel.

Les princes en exil ne sont pratiquement pas pris en compte dans les sources russes. Seule la mort de Dmitrij Šemjaka, empoisonné à Novgorod par un agent de Vasilij II, est chroniquée, de façon plus ou moins édulcorée, parce qu'il s'agissait d'un rival extrêmement dangereux qui demeurait encore dans l'espace russe ${ }^{39}$. Les autres princes émigrés se perdent dans l'oubli dès qu'ils franchissent la frontière de la Pologne-Lituanie. Les écrits d'Ivan le Terrible peuvent toutefois nous donner un indice de la façon dont ils étaient perçus. Dans sa première lettre à Andrej Kurbskij (1564), le tsar considère ce prince, descendant de la lignée de Jaroslavl' , comme un renégat qui a trahi son maître et sa foi pour s'allier avec les ennemis de la chrétienté, et le compare aux empereurs iconoclastes, Léon III l'Isaurien, Constantin Copronyme et Léon V l'Arménien. Il est donc clair que Kurbskij est voué à la damnation $^{40}$. Dans son testament de 1572, Ivan IV se dépeint lui-même comme « un exilé,

34. PSRL, t. 1, col. 313-318 ; t. 2, col. 321-327, 337, 349-355 et M. Dimnik, The Dynasty of Chernigov, 1146-1246, Cambridge, 2003, p. 35, 45-48.

35. PSRL, t. 1, col. 426.

36. PLDR, t. 4,p. 516.

37. PSRL, t. 12, p. 214 (Vasilij Jaroslavič, $\mathrm{n}^{\circ}$ 6.13, « mort dans les chaînes ») ; t. 13, p. 11 (Dmitrij, petit-fils d'Ivan III, 9.1).

38. PSRL, t. 13, p.115 (Jurij Ivanovič, 8.3), 121 (Andrej Ivanovič, 8.6).

39. PSRL, t. 20.1 , p. $262 ;$ t. 23 , p. 155.

40. PLDR, t. 8, Vtoraja polovina XVI veka, M., 1986, p. 24. 
du fait de l'indocilité des boyards, contraint d'errer de contrée en contrée ${ }^{41}$. Cette phrase est ambiguë : Ivan se considère-t-il lui aussi comme un «maudit», à l'image de Svjatopolk, l'assassin de Boris et Gleb qui mourut en terre étrangère après une vaine errance, ou bien pense-t-il subir un exil purificateur, comme Israël en Egypte ? ${ }^{42}$ Dans ce cas, les autres exilés de la dynastie contribueraient eux aussi, à leur façon, à sa sanctification.

Nous avons donc rencontré les princes qui quittent le monde pour le cloître, la prison ou l'exil, et les princes qui se doivent d'y demeurer pour accomplir jusqu'au bout leur fonction. Il existe toutefois une position intermédiaire, au moins pour les cadets de la dynastie.

\section{Le prince « nourricier » : trépas édifiants et legs pieux}

À priori, tout prince qui se respecte devrait entrer dans la catégorie des « nourriciers » $(\text { kormiteli })^{43}$, tant la générosité envers le clergé fait partie intégrante du portrait du bon prince. Toutefois, nous l'avons vu, les chefs de la famille moscovite calculent leurs libéralités envers les monastères. Dès la génération de Dmitrij Donskoj, les «nourriciers » se trouvent donc parmi les cadets de la dynastie, à condition qu'ils conservent la capacité d'agir ; nous verrons d'ailleurs que leurs dispositions testamentaires étaient toujours susceptibles d'être invalidées ou modifiées par le souverain.

Le premier est le cousin et auxiliaire de Dmitrij, Vladimir Andreevič de Serpuhov-Borovsk qui «aimait les monastères et rendait un grand honneur à l'ordre monastique $»^{44}$. Au-delà de cette formule, on sait que Vladimir eut à cœur de doter la capitale de sa principauté, Serpuhov, d'un monastère dont le fondateur fut Serge de Radonež qui baptisa aussi son fils Ivan (5.9) ; c'est d'ailleurs sur sa principauté que se trouvait le monastère de la Trinité ${ }^{4}$. Après sa mort, son petit-fils Vasilij Jaroslavič fut aussi proche de l'abbaye, mais leurs relations durent cesser lorsqu'il fut arrêté par Vasilij II, en $1454^{46}$.

41. «изгнан есмь от бояр, самоволства их ради, от своего достояния и скитаюся по странам », DDG, $\mathrm{n}^{\circ} 104$, p. 427.

42. Sur la mort de Svjatopolk, voir PLDR, t. 1, p. 158-160; on notera que lorsqu'en 1584 Ivan demande aux moines russes de prier pour lui, il se qualifie de «maudit» (чтоб вы пожаловали, о моем окаянстве соборно и по кельям молили Бога), DAI, t. 1, n 129 , p. 185 .

43. Ce qualificatif est employé par Cyrille de Beloozero lui-même, dans le récit d'un de ses miracles posthumes, voir G. M. Prohorov, éd., Prepodobnye Kirill, Ferapont i Martinian Belozerskie, SPb., 1993, p. 154 (Drevnerusskie skazanija o dostopamjatnyh ljudjah, mestah i sobytijah, 1).

44. PSRL,t. 18 ,p. 114 ; t. 11 ,p. 20.

45. PSRL,t. 18, p. 114, 131 ; t. 11, p. 20,70 ; PLDR, t. 4, p. 390 ; P. Gonneau, La Maison de la Sainte Trinité..., op. cit., p. 131-132, 139.

46. P. Gonneau, La Maison de la Sainte Trinité..., op. cit., p. 162, 171-173,182. 
Sous les règnes de Vasilij Irer et Vasilij II, la branche cadette de Galič montre aussi une forte dévotion envers le monastère de Serge. Jurij Dmitrievič (5.3), qui avait été baptisé par le saint abbé, assista à l'invention de ses reliques en 1422 et finança alors la construction de l'abbatiale en pierre de la Trinité ${ }^{47}$. L'un de ses fils, Dmitrij le Bel (6.9), légua un important village au monastère ${ }^{48}$. Son trépas, survenu en 1440, dans une période d'accalmie pendant les guerres dynastiques qui déchiraient la Moscovie, a fait l'objet d'un récit particulier qui suggère qu'il mourut en odeur de sainteté 49 .

La branche de Beloozero-Vereja, issue d'Andrej Dmitrievič (5.6), entretint pour sa part des liens privilégiés avec les monastères Saint-Cyrille de Beloozero et SaintPaphnuce de Borovsk. Les textes hagiographiques célébrant Cyrille, Théraponte et Martinien de Beloozero attestent qu'une relation étroite se noua du vivant d'Andrej Dmitrievič et que la femme de Mihail Andreevič fut guérie d'une infirmité à la jambe par un miracle posthume de Cyrille ${ }^{50}$. Mihail Andreevič (6.11) était le dernier survivant de la génération de Vasilij II lorsqu'il mourut, en 1485. Il dut réviser à plusieurs reprises son testament, sur les injonctions d'Ivan III. Malgré tout, dans son ultime version, il prévoit d'assez nombreux legs aux monastères de Beloozero, ainsi qu'à des fondations moscovites ${ }^{51}$. Le prince se fit enterrer à SaintPaphnuce de Borovsk, abbaye d'un saint intransigeant qui s'était même opposé à l'autocéphalie de l'Église russe ${ }^{52}$.

Les trois des cadets d'Ivan III qui conservèrent leur liberté (Jurij, Andrej le Cadet et Boris) se montrèrent autant que possible proches des monastères et généreux envers eux jusqu'au bout. Jurij (7.3), mort le premier, sans enfants, effectua une impressionnante série de legs pieux à diverses fondations. Le récit de ses funérailles insiste sur la profondeur du deuil qui frappa la foule à sa mort. On peut voir dans cette notation un signe de l'attachement des milieux religieux au prince ${ }^{53}$. Andrej le Cadet (7.7) prévoyait aussi de céder un domaine conséquent à la Trinité Saint-Serge et de gratifier «son » monastère du Sauveur à Kamen’e, sur le lac Kubenskoe. Toutefois son testament fut cassé, soit parce qu'on le soupçonnait d'avoir été falsifié, soit parce qu'il déplut à Ivan III $^{54}$. De Boris Vasil'evič (7.6),

47. PSRL, t. 18, p. 115 ; B. M. Kloss, Žitie Sergija Radonežskogo, M., 1998, p. 418-419 (Izbrannye trudy, 1) ; P. Gonneau, La Maison de la Sainte-Trinité..., op. cit., p. 131, 139, 152153.

48. ASEI, t. $1, \mathrm{n}^{\circ} 164$.

49. PSRL, t. 27, p. 107-108 ; P. Gonneau, «L'ideale della santità monastica... », art. cit., p. 113-114, 124-125.

50. Voir G. M. Prohorov, éd., Prepodobnye Kirill, Ferapont i Martinian Belozerskie..., op. cit., p. $99,154,220,246,319$.

51. $D D G, \mathrm{n}^{\circ} 80$.

52. PSRL, t. 12, p. 218 ; voir aussi le Récit sur la vie de Paphnuce dans PLDR, t. 5 : Vtoraja polovina $X$ V veka, M., 1982,p. 478-513.

53. DDG $\mathrm{n}^{\circ} 68$; PSRL, t. 25, p. 298 ; P. Gonneau, «L'ideale della santità monastica... », art. cit.,p. 109-110, 127.

54. $D D G, \mathrm{n}^{\circ} 74$; PSRL, t. 18 , p. 269 ; t. 26 , p. 274 ; t. 23 ,p. $163,186$. 
prince de Volokolamsk et Ruza, on ne conserve qu'un testament de 1477, bien antérieur à sa mort, en $1494^{55}$. Il précède également la fondation du monastère de la Dormition, près de Volokolamsk, par Joseph Sanin (1479). Cette abbaye devint rapidement un des phares du monachisme russe. Boris et ses descendants entretinrent des relations parfois orageuses avec Joseph et ses moines. À la mort de Boris, son corps fut ramené à Moscou, sur ordre d'Ivan III. En revanche, ses deux fils, Fedor (8.9) et Ivan (8.10), reposent en l'abbatiale de la Dormition Saint-Joseph ${ }^{56}$.

Le dernier cas de «prince-nourricier » est exemplaire. Dmitrij Ivanovič (8.4), frère cadet de Vasilij III, attribuait dans son testament des dons à une quarantaine de monastères et d'églises, en particulier celles dédiées à son saint patron Démétrios, à la condition que le grand-prince y consente ${ }^{57}$. Cette générosité ne suffit pas à expliquer pourquoi la Chronique de la Typographie, une compilation connue pour sa relative liberté de ton, consacre un long récit à son trépas et à ses funérailles $(1521)^{58}$. À la lecture de ce texte, qui n'est pas repris dans les chroniques moscovites, Dmitrij semble avoir joui d'une grande popularité dans sa propre principauté, mais aussi dans les milieux religieux, peut-être parce qu'il avait pu incarner une sorte de recours acceptable, tempérant l'autoritarisme de son frère aîné ? On peut penser que le texte a été rédigé à la Trinité Saint-Serge, parce qu'il évoque assez précisément le passage du convoi funéraire en cette abbaye ; en outre, la Chronique de la Typographie comporte dans ces années-là des informations précises ne concernant que l'abbaye de la Trinité59. Le plus frappant dans ce récit est de voir que, face à l'attachement des foules, qui vouent une vénération spontanée au prince, veulent toucher son cercueil, comme celui d'un thaumaturge, et garder sa dépouille, les autorités moscovites n'ont qu'un souci : ramener le corps à Moscou pour mettre fin à ces épanchements inquiétants. Le boyard Semen Ivanovič Voroncov a bien du mal à se faire remettre la dépouille et à congédier les fidèles : « Si Semen ne l'avait pas interdit, tout le peuple l'aurait suivi jusqu'à la ville impériale de Moscou ». En 1610, en plein Temps des Troubles, le tsar Vasilij Šujskij ordonna de la même façon de ramener à Moscou la dépouille du tsarévitch Dmitrij qui reposait à Uglič depuis neuf ans. Il s'agissait de renforcer le "pôle de sainteté » de la collégiale SaintMichel du kremlin et aussi d'éviter qu'un culte local servît de point de ralliement aux adversaires de l'ordre moscovite.

Il est incontestable que le trépas et le destin posthume des fils de la lignée moscovite révèlent à la fois des comportements religieux, des calculs politiques et certaines conceptions (car elles peuvent varier) de la fonction princière. Ces observations ponctuelles aboutissent-elles à un tableau cohérent?

\section{5. $D D G, \mathrm{n}^{\circ} 71$.}

56. PSRL, t. 12 , p. 238,258 ; t. 13, p. 16 ; P. Gonneau, La Maison de la Sainte Trinité..., op. cit., p. 208, 215-217.

57. $D D G, \mathrm{n}^{\circ} 99$.

58. PSRL, t. 24, p. 218-220 ; P. Gonneau, «L'ideale della santità monastica... », art. cit., p. $112,114-118,130-132$.

59. A. N. Nasonov, Istorija russkogo letopisanija XI-načala XVIII vV., M., 1969, p. 388-392. 


\section{Essai d'interprétation}

Dans un récent article, S. V. Sazonov distingue deux attitudes face à la mort dans la culture russe médiévale. Les mentalités de la période de Kiev sont marquées par une « confiance tranquille » quant au salut, fondée sur l'opposition simple entre les chrétiens, destinés à être sauvés, et les païens que seule la conversion peut racheter. Les défunts morts dans la religion orthodoxe reposent forcément parmi les justes et les vivants peuvent compter sur leur intercession. Mais, à partir de la fin du XII ${ }^{\mathrm{e}}$ siècle et surtout à la suite de l'invasion mongole, l'angoisse de la damnation devient plus forte. Les châtiments envoyés par Dieu montrent que la distinction fondamentale n'est pas entre les chrétiens et les païens, mais entre les bons et les mauvais chrétiens. Il paraît dès lors nécessaire de se munir de garanties nouvelles, comme la prise d'habit, pour un salut personnel et non collectif. Désormais aussi, les morts ont besoin des prières et de la commémoration des vivants. La vision pessimiste l'emporte jusqu'à la fin du XIVe siècle où la redécouverte de «l'antiquité » kiévienne par les lettrés rétablit la confiance, au moins dans l'entourage de Dmitrij Donskoj. Toutefois, un parti emmené par la haute hiérarchie ecclésiastique, maintient que l'appartenance au peuple chrétien ou même à la lignée de saint Vladimir ne suffit pas à éviter la damnation $^{60}$. Il nous semble que cette présentation binaire ne permet pas, à elle seule, d'expliquer les comportements que nous rencontrons dans la dynastie moscovite.

Nous avons, pour notre part, observé trois types d'attitudes face à la mort et au salut : le prince-moine, le prince séculier et le prince nourricier. Mais peut-être estil plus révélateur de classer les individus selon la fonction qu'ils exerçaient au moment du trépas, car elle détermine fortement leur choix.

Les aînés de la lignée moscovite, c'est-à-dire les souverains et les héritiers désignés, privilégient le trépas monastique, d'Aleksandr Nevskij à Ivan II le Bel. Ils l'ont choisi par fidélité à l'exemple d'Aleksandr, mais aussi parce que leur famille, contrairement à celle de Tver' par exemple, ne compte aucun prince-martyr, sanctifié par ses souffrances, et doit donc adopter un autre modèle ${ }^{61}$. Les aînés de Moscou optent pour un trépas séculier à partir du règne de Dmitrij Donskoj, au moment où ils confisquent à leur profit la fonction de grand-prince de Vladimir et aussi au moment où le principe de la succession verticale se substitue à celui de la succession horizontale, traditionnel jusque-là. À Dmitrij Donskoj succède Vasilij I Ir son fils, puis le fils de ce dernier, Vasilij II ${ }^{62}$. Ce dernier doit lutter contre

60. S. V. Sazonov, «K probleme vosprijatii smerti v srednevekovoj Rusi», in Russkaja istorija : problemy mentaliteta, M., 1994, p. 47-52.

61. Jurij Danilovič mourut de mort violente à la Horde (1325), mais il avait auparavant été le complice du martyre du prince Mihail Jaroslavič de Tver' (1318); en outre, il fut assassiné par le fils de Mihail de Tver' et non exécuté sur l'ordre du khan, voir PSRL, t. 15, col. 412, 415 ; t. 15.1, col. 40-42. ; t. 25, p. 161-167.

62. Vladimir Andreevič $\left(n^{\circ} 4.10\right)$ ne pouvait en principe occuper le trône aîné, parce que son père, Andrej ( $\left.n^{\circ} 3.4\right)$, était mort de la peste en 1353 sans avoir régné à Moscou. Il y eut toutefois une période de brouille entre lui et Vasilij Ir au début du règne de ce dernier, voir PSRL, t. 25, p. 218. Sur la guerre dynastique de 1425-1453, voir en particulier A. A. Zimin, Vitjaz' na rasput'e : feodal'naja vojna v Rossii XV v., M., 1991. 
son oncle et ses cousins, mais, après sa victoire, la transmission du pouvoir se fait selon la primogéniture mâle. Dès lors, l'aîné de la dynastie ne peut abandonner sa position sans risquer de déstabiliser l'édifice de la monarchie. Si Vasilij III et Ivan le Terrible reviennent au modèle de trépas monastique, c'est certainement parce qu'ils doutent de leur salut, mais aussi parce que leur entourage se convainc que leur fin est véritablement imminente.

Les princes cadets qui exercent pleinement leurs fonctions, en assistant leur aîné et en gouvernant leur propre territoire, sont d'abord peu enclins à opter pour le trépas monastique : avant 1389, seul Afanasij Danilovič (2.4) choisit cette mort. Entre la fin du XIVe siècle et les années $30 \mathrm{du} \mathrm{XV}^{\mathrm{e}}$ siècle, dans une période marquée par la mort de Serge de Radonež et l'invention de ses reliques (1392, 1422), le raid tatar d'Edigej (1408) et plusieurs épidémies de peste, on compte trois vocations, chez des princes obscurs $\left(n^{\circ} 5.5,5.10,6.6\right)$. Mais le type du prince-nourricier est plus caractéristique des princes cadets. Il apparaît lui aussi à la fin du XIVe siècle et se développe particulièrement sous les règnes de Vasilij II, d'Ivan III et de Vasilij III. C'est qu'entretenir des relations privilégiées avec une ou plusieurs abbayes est, pour un cadet, une façon acceptable d'exprimer ses préoccupations spirituelles, d'exercer ses prérogatives temporelles et de défendre l'identité locale de son territoire. En outre, l'intercession d'une puissante communauté monastique peut, éventuellement, lui éviter la disgrâce du souverain moscovite. On remarque que les princes cadets devenus moines ou figurant parmi les «nourriciers » sont les plus susceptibles de ne pas être ensevelis aux côtés de leurs frères et cousins en la collégiale Saint-Michel du kremlin. Est-ce à cause de leur proximité particulière avec l'ordre monastique ou parce qu'ils sont suffisamment effacés pour que l'on juge inutile de rapatrier leur dépouille dans la nécropole familiale?

Les princes cadets privés de l'exercice de leur fonction ont des choix très limités et même la possibilité de devenir moine ne leur est laissée qu'avec réticences. Ceux qui meurent en prison se voient reconnaître le statut de princes souffrants, sinon de véritables martyrs, et obtiennent souvent d'être ensevelis dans la nécropole familiale, ce qui est une sorte de réhabilitation posthume. Ceux qui ont préféré l'exil à la prison sont réputés mourir en état d'errance. Mais même ainsi, tout lien ne paraît pas rompu entre eux et la lignée, car un retour est toujours envisageable. Dmitrij Šemjaka (6.8) et son fils Ivan (7.8) moururent en exil, mais le petit-fils, Vasilij Ivanovič (8.11), fut admis à reprendre son rang dans la famille régnante lorsqu'il quitta la Lituanie, en 1500. Cette réconciliation fut toutefois précaire, car il fut arrêté en 1523 et mourut en prison ${ }^{63}$.

La solidarité lignagère, symbolisée à Moscou par la nécropole de l'archange Saint-Michel, demeure le fondement de l'espérance dans le salut. Certes, les vivants prient plus que jamais pour les morts, au fur et à mesure que Moscou affirme sa puissance, mais ils comptent aussi sur l'aide de leurs ascendants et pas seulement de ceux que l'Église vénère officiellement, comme Vladimir, Boris et Gleb ou Aleksandr Nevskij. Gail D. Lenhoff a insisté à juste titre sur le fait que le 
principal monument de l'historiographie moscovite du milieu du XVI siècle, le Livre des degrés de la généalogie impériale, esquisse un programme de vénération collective de la dynastie moscovite ${ }^{64}$. Cette tentative n'aurait rien d'exceptionnel, puisque plusieurs monarchies ont poursuivi ce but, avec des succès divers ${ }^{65}$. Les efforts russes n'aboutissent pas, sans doute en grande partie à cause de l'échec spirituel que fut le règne d'Ivan le Terrible aux yeux de l'Église, alors que les commanditaires de la Stepennaja kniga comptaient en faire le 17e et ultime degré de l'ascension mystique du peuple russe vers le Salut. Significativement, ce degré est inachevé et toutes les velléités de poursuivre et de conclure le Livre des degrés sont demeurées vaines. Il n'en reste pas moins que la sainteté, comme la légitimité dynastique, est descendante et non ascendante. Elle procède du premier degré, Vladimir, et doit se transmettre à chaque génération pour être encore efficace. De même qu'un prince dont le père n'a pas régné sur le trône de Kiev (ou de Moscou) ne saurait légitimement y prétendre, fût-il un descendant en droite ligne de Vladimir, de même un prince dont le père n'aurait pas été, lui aussi, sanctifié, ne pourrait se prévaloir de la sainteté de Vladimir.

En définitive, quelle que soit la dévotion personnelle et la situation matérielle du prince, il ne saurait être totalement coupé de son arbre généalogique. La métaphore de l'arbre est précisément celle qui ouvre le Livre des degrés et constitue son principe d'organisation. Dans la préface de l'ouvrage, la descendance de Vladimir est définie comme une « lignée de justes » (semeni ego pravednago) où chacun «plaît à Dieu » (Bogu ugodiša) à sa façon. Les uns le font

en régnant heureusement, en vivant dans le mariage, en assurant une noble et nombreuse descendance; les autres dans le célibat, par la pureté, le monachisme, le martyre pour le Christ, par la bravoure dans les batailles, par une patience reconnaissante dans la captivité, les malheurs, les chaînes, les prisons, en subissant, lors des luttes intestines, les avanies, l'aveuglement, la réclusion ; d'autres encore, en acceptant librement la pauvreté et l'errance, en feignant la folie qui est sagesse divine, en vivant sans domicile dans leur pays et à l'étranger, ou grâce à d'autres vertus encore $[\ldots]^{66}$.

64. «Unofficial Veneration of the Daniilovichi in Muscovite Rus'», in A. M. Kleimola, G. Lenhoff, éds., Culture and Identity in Muscovy, 1359-1584 = Moskovskaja Rus' (13591584) : kul'tura i istoričeskoe samosoznanie, M., 1997, p. 391-416, en particulier p. 414-416.

65. G. Klaniczay, « From Sacral Kinship to Self-Representation : Hungarian and European Royal Saints in the XI-XIIIth Centuries », in E. Vestergaard ,éd., Continuity and Change in the Middle Ages, Odense, 1986, p.61-86 ; A. Vauchez, "'Beata stirps' : sainteté et lignage en Occident aux XIII et XIVe siècles », in Famille et parenté dans l'Occident médiéval, Rome, 1977, p. 397-406 (Collection de l'Ecole française de Rome, 30).

66. «въ благоденственномъ державствъ, въ супружествъ живуще и во благородномъ многочадіи ; овіи же безсупружествомъ, чистотою, иночествомъ и мученіемъ за Христа, и на бранех храбростію, и благодарнымъ терпьніемъ во плененіихъ, въ нужахъ, и вь юзахъ, и въ темницахъ, и въ межеусобныхъ крамолахъ озлобленіемъ и лишеніемъ очію и заточеніемъ, и ини же самоволною нищетою и странствіемъ и богомудростнымъ претворенія уродствомъ, и бездомствіемъ во отечествіихъ и въ чюжеземствіихъ и прочими добродътельми », PSRL, t. 21.1, p. 5. 
Cette paraphrase inspirée de Paul (Rm. 12 :4-8) montre que les fonctions des princes sont variées, mais qu'ils forment un seul corps mystique de la royauté, qu'ils exercent tous un même sacerdoce royal.

On trouve là une conception qui nous rappelle les théologies politiques élaborées en Occident, particulièrement en Angleterre et en France, sur la continuité dynastique et les deux corps du roi. À l'Ouest aussi, « il est clair que la doctrine de la théologie et du droit canon, qui enseigne que l'Église et la société chrétienne en général sont un corpus mysticum, dont la tête est le Christ, a été transportée par les juristes de la sphère théologique à celle de l'État, dont la tête est le roi » ${ }^{67}$. Or, selon la doctrine thomiste, tandis que dans le corps humain les membres sont présents « tous à la fois », dans le corps mystique du Christ, les membres s'accumulent petit à petit, en une succession permanente, « depuis le début du monde jusqu'à la fin du monde ». Dès lors, aux yeux des théoriciens, « la dynastie, la "maison”, ressemblait à une somme supra-individuelle, comparable à une universitas qui "ne mourait jamais" ${ }^{68}$. Il y a toutefois une différence significative. En Occident, dans la succession dynastique, comme au sein des corporations,

on construisait une personne corporative, une sorte de personna mystica, qui n'était une collectivité que sur le plan du temps, puisque la pluralité de ses membres était formée uniquement par la succession [...], on construisait un corps dont les membres s'échelonnaient en longueur, si bien que sa coupe à tout moment révélait un seul membre ${ }^{69}$.

Il nous semble que, de son côté, la dynastie moscovite, constitue bien un corps mystique par accumulation successive dans le temps ; toutefois, à chaque génération, ce corps n'est pas formé seulement par le souverain en exercice, mais par la collectivité, ou la corporation qu'il constitue en compagnie de ses frères et cousins. La corporation se matérialise de façon éclatante dans la nécropole familiale « où reposent tous les pieux grands-princes, leur lignée $»^{70}$.

École pratique des hautes études

Sciences historiques et philologiques

Université de Paris-Sorbonne (Paris IV)

pierre.gonneau@wanadoo.fr

67. E. Kantorowicz, Les deux corps du roi : essai sur la théologie politique au Moyen âge, Paris, 1989, p. 28 (Bibliothèque des histoires).

68. Ibid., p. 223, 243.

69. Ibid., p. 227.

70. « Идъ же вси благовърнии велиции князи лежат, род их », PSRL, t. 25, p. 298. 
Les trépas des princes de la dynastie moscovite

\begin{tabular}{|c|c|c|c|c|c|}
\hline nom (gd-prince) & père (numéro) & $\begin{array}{c}\text { date } \\
\text { de mort }\end{array}$ & conditions & $\begin{array}{l}\text { testa- } \\
\text { ment }\end{array}$ & sépulture \\
\hline-2 Jaroslav & Vsevolod & 1246 & $\mathrm{~L}(\mathrm{H})$ & - & Vladimir, cathédrale \\
\hline - 1 Aleksandr Nevskij & Jaroslav (-2) & 1263 & $\mathbf{M}(\mathrm{H})$ & - & Vladimir, cathédrale \\
\hline 1 Daniil & Aleksandr (-1) & 1303 & $\mathrm{M}$ & - & Moscou, St-Daniel \\
\hline 2.1 Jurij & Danil (1) & 1325 & $\mathrm{~L}(\mathrm{H})$ & - & Moscou, cathédrale \\
\hline 2.2 Aleksandr & Danil (1) & 1308 & $\mathrm{~L}$ & - & Tver'? \\
\hline 2.3 Boris & Danil (1) & 1320 & $\mathrm{~L}$ & - & Vladimir, cathédrale \\
\hline 2.4 Afanasij & Danil (1) & 1322 & $\mathrm{M}$ & - & Novgorod, Sauveur \\
\hline 2.5 Ivan Ier Kalita & Danil (1) & 1341 & $\mathrm{M}$ & $\mathrm{T}$ & Moscou, St-Michel \\
\hline 3.1 Semen Le Fier & Ivan Ier (2.5) & 1353 & $\mathrm{~L}$ (peste) & $\mathrm{T}$ & Moscou, St-Michel \\
\hline 3.2 Daniil & Ivan Ier (2.5) & $1320 \mathrm{v}$ & $\mathrm{L}(\min )$. & - & $?$ \\
\hline 3.3 Ivan II Le Bel & Ivan I ${ }^{\mathrm{er}}(2.5)$ & 1359 & $\mathrm{M}$ & $\mathrm{T}$ & Moscou, St-Michel \\
\hline 3.4 Andrej & Ivan I ${ }^{\mathrm{er}}(2.5)$ & 1353 & $\mathrm{~L}$ (peste) & - & Moscou, St-Michel \\
\hline 4.1 Vasilij & Semen (3.1) & 1338 & $\mathrm{~L}(\min )$. & - & $?$ \\
\hline 4.2 Konstantin & Semen (3.1) & 1341 & $\mathrm{~L}(\min )$. & - & $?$ \\
\hline 4.3 Daniil & Semen (3.1) & $1347 \mathrm{v}$ & $\mathrm{L}(\min )$. & - & $?$ \\
\hline 4.4 Mihail & Semen (3.1) & $1348 \mathrm{v}$ & $\mathrm{L}(\min )$. & - & $?$ \\
\hline 4.5 Ivan & Semen (3.1) & 1353 & $\mathrm{~L}(\min )$. & - & $?$ \\
\hline 4.6 Semen & Semen (3.1) & 1353 & $\mathrm{~L}$ (min.) & - & $?$ \\
\hline 4.7 Dmitrij Donskoj & Ivan II (3.3) & 1389 & $\mathrm{~L}$ & $2 \mathrm{~T}$ & Moscou, St-Michel \\
\hline 4.8 Ivan & Ivan II (3.3) & 1364 & $\mathrm{~L}$ & - & Moscou, St-Michel \\
\hline 4.9 Ivan & Andrej (3.4) & 1368 & $\mathrm{~L}$ & - & $?$ \\
\hline 4.10 Vladimir & Andrej (3.4) & 1410 & $\mathrm{~L}$ & $\mathrm{~T}$ & Moscou, St-Michel \\
\hline 5.1 Daniil & Dmitrij (4.7) & $?$ & $\mathrm{~L}$ (min.) & - & $?$ \\
\hline 5.2 Vasilij Ier & Dmitrij (4.7) & 1425 & $\mathrm{~L}$ & $3 \mathrm{~T}$ & Moscou, St-Michel \\
\hline 5.3 Jurij & Dmitrij (4.7) & 1434 & $\mathrm{~L}$ & $\mathrm{~T}$ & Moscou, St-Michel \\
\hline 5.4 Semen & Dmitrij (4.7) & 1379 & $\mathrm{~L}$ & - & $?$ \\
\hline 5.5 Ivan / Josaphat & Dmitrij (4.7) & 1393 & $\mathrm{M}$ & - & Moscou, Sauveura \\
\hline 5.6 Andrej & Dmitrij (4.7) & 1432 & $\mathrm{~L}$ & - & Moscou, St-Michel \\
\hline 5.7 Petr & Dmitrij (4.7) & 1428 & $\mathrm{~L}$ & - & Moscou, St-Michel \\
\hline 5.8 Konstantin & Dmitrij (4.7) & $1433 ?$ & $\mathrm{~L}$ & - & $?$ \\
\hline 5.9 Ivan & Vladimir (4.10) & 1422 & $\mathrm{~L}$ & - & Moscou, St-Michel \\
\hline 5.10 Semen / Sabas & Vladimir (4.10) & 1426 & $\mathbf{M}$ (peste) & - & Trinité St-Serge \\
\hline 5.11 Andrej & Vladimir (4.10) & $?$ & $\mathrm{~L}(\min )$. & - & $?$ \\
\hline 5.12 Jaroslav & Vladimir (4.10) & 1426 & $\mathrm{~L}$ (peste) & - & Moscou, St-Michel \\
\hline 5.13 Fedor & Vladimir (4.10) & 1390 ap. & $\mathrm{L}(\min )$. & - & $?$ \\
\hline 5.14 Andrej & Vladimir (4.10) & 1426 & $\mathrm{~L}$ (peste) & - & Moscou, St-Michel \\
\hline 5.15 Vasilij & Vladimir (4.10) & 1427 & $\mathrm{~L}$ & - & Moscou, St-Michel \\
\hline
\end{tabular}

$\mathrm{H}=$ mort à la Horde, où en rentrant de la Horde, $\mathrm{L}=$ Laïc, $\mathrm{M}=$ moine, min. = mineur Les grands-princes ou tsars apparaissent en caractères gras.

a. Monastère des femmes de la dynastie, le prince repose auprès de sa grand-mère. 


\begin{tabular}{|c|c|c|c|c|c|}
\hline nom (gd-prince) & père (numéro) & $\begin{array}{c}\text { date } \\
\text { de mort }\end{array}$ & conditions & $\begin{array}{l}\text { testa- } \\
\text { ment }\end{array}$ & sépulture \\
\hline 6.1 Jurij & Vasilij $1^{\mathrm{e}}(5.2)$ & 1400 & $\mathrm{~L}(\min )$. & - & Moscou, St-Michel \\
\hline 6.2 Ivan & Vasilij 1e (5.2) & 1417 & $\mathrm{~L}(\min )$. & - & Moscou, St-Michel \\
\hline 6.3 Daniil & Vasilij I Ir (5.2) & $1402 ?$ & $\mathrm{~L}(\min )$. & - & $?$ \\
\hline 6.4 Semen & Vasilij I I & $1405 ?$ & $\mathrm{~L}(\min )$. & - & $?$ \\
\hline 6.5 Vasilij II & Vasilij I I ${ }^{e r}$ (5.2) & 1462 & $\mathrm{~L}$ & $\mathrm{~T}$ & Moscou, St-Michel \\
\hline 6.6 Ivan / Ignace & Jurij (5.3) & 1432 & $\mathbf{M}$ & - & Galič, Sauveur \\
\hline 6.7 Vasilij & Jurij (5.3) & 1448 & $\mathrm{~L}$ (prison) & - & Moscou, St-Michel \\
\hline 6.8 Dmitrij Šemjaka & Jurij (5.3) & 1453 & L (exil) & - & Novgorod, St-Georges \\
\hline 6.9 Dmitrij le Bel & Jurij (5.3) & 1440 & $\mathrm{~L}$ & $\mathrm{~b}$ & Moscou, St-Michel \\
\hline 6.10 Ivan & Andrej (5.6) & 1462 & L (exil) & - & $?$ \\
\hline 6.11 Mihail & Andrej (5.6) & 1485 & $\mathrm{~L}$ & $\mathrm{~T}$ & Borovsk, St-Paphnuce \\
\hline 6.12 Semen & Konstantin (5.8) & $?$ & $\mathrm{~L}$ (min. ?) & - & $?$ \\
\hline 6.13 Vasilij & Jaroslav (5.12) & 1483 & $\mathrm{~L}$ (prison) & - & Moscou, St-Michel \\
\hline 7.1 Jurij & Vasilij II (6.5) & 1441 & $\mathrm{~L}(\min )$. & - & $?$ \\
\hline 7.2 Ivan III & Vasilij II (6.5) & 1505 & $\mathrm{~L}$ & $\mathrm{~T}$ & Moscou, St-Michel \\
\hline 7.3 Jurij & Vasilij II (6.5) & 1472 & $\mathrm{~L}$ & $\mathrm{~T}$ & Moscou, St-Michel \\
\hline 7.4 Andrej 1'Aîné & Vasilij II (6.5) & 1493 & $\mathrm{~L}$ (prison) & - & Moscou, St-Michel \\
\hline 7.5 Semen & Vasilij II (6.5) & $?$ & $\mathrm{~L}$ (min. ?) & - & $?$ \\
\hline 7.6 Boris & Vasilij II (6.5) & 1494 & $\mathrm{~L}$ & $\mathrm{~T}$ & Moscou, St-Michel \\
\hline 7.7 Andrej le Cadet & Vasilij II (6.5) & 1481 & $\mathrm{~L}$ & $\mathrm{~T}$ & Moscou, St-Michel \\
\hline 7.8 Ivan & Dmitrij (6.8) & 1485 ap. & $\mathrm{L}$ (exil) & - & $?$ \\
\hline 7.9 Andrej & $\operatorname{Ivan}(6.10)$ & $?$ & L (exil) & - & $?$ \\
\hline 7.10 Semen & $\operatorname{Ivan}(6.10)$ & $?$ & L (exil) & - & $?$ \\
\hline 7.11 Vasilij & Mihail (6.11) & 1495 & $\mathrm{~L}$ & - & $?$ \\
\hline 7.12 Ivan & Mihail (6.11) & $1485 \mathrm{v}$ & $\mathrm{L}$ & - & $?$ \\
\hline 7.13 Ivan & Vasilij (6.13) & $?$ & L (exil) & - & $?$ \\
\hline 7.14 Ivan & Vasilij (6.13) & $?$ & $\mathrm{~L}$ (prison) & - & $?$ \\
\hline 7.15 Andrej & Vasilij (6.13) & $?$ & $\mathrm{~L}$ (prison) & - & $?$ \\
\hline 7.16 Vasilij & Vasilij (6.13) & $?$ & $\mathrm{~L}$ (prison) & - & $?$ \\
\hline 8.1 Ivan & Ivan III (7.2) & 1490 & $\mathrm{~L}$ & - & Moscou, St-Michel \\
\hline 8.2 Vasilij III /Barlaam & Ivan III (7.2) & 1533 & $\mathbf{M}$ & $\mathrm{T}$ & Moscou, St-Michel \\
\hline 8.3 Jurij & Ivan III (7.2) & 1536 & $\mathrm{~L}$ (prison) & - & Moscou, St-Michel \\
\hline 8.4 Dmitrij & Ivan III (7.2) & 1521 & $\mathrm{~L}$ & $\mathrm{~T}$ & Moscou, St-Michel \\
\hline 8.5 Semen & Ivan III (7.2) & 1518 & $\mathrm{~L}$ & - & Moscou, St-Michel \\
\hline 8.6 Andrej & Ivan III (7.2) & 1537 & $\mathrm{~L}$ (prison) & - & Moscou, St-Michel \\
\hline 8.7 Ivan / Ignace, st & $\begin{array}{l}\text { Andrej 1'Aîné } \\
(7.4)\end{array}$ & $1523 ?$ & $\mathbf{M}$ (prison) & - & Sauveur de Priluki \\
\hline 8.8 Dmitrij & $\begin{array}{l}\text { Andrej l'Aîné } \\
(7.4)\end{array}$ & $1550 \mathrm{v}$ & $\mathrm{L}$ (prison) & - & $?$ \\
\hline
\end{tabular}

b. Pas de testament conservé, mais une donation en exécution du testament du prince, voir $A S E I, \mathrm{t} .1, \mathrm{n}^{\circ} 164$. 


\begin{tabular}{|l|l|c|c|c|l|}
\hline \multicolumn{1}{|c|}{ nom (gd-prince) } & père (numéro) & $\begin{array}{c}\text { date } \\
\text { de mort }\end{array}$ & conditions & $\begin{array}{c}\text { testa- } \\
\text { ment }\end{array}$ & \multicolumn{1}{|c|}{ sépulture } \\
\hline 8.9 Fedor & Boris (7.6) & 1513 & L & T & Volok, St-Joseph \\
\hline 8.10 Ivan & Boris (7.6) & 1504 & L & T & Volok, St-Joseph \\
\hline 8.11 Vasilij & Ivan (7.8) & 1529 & L (prison) & - & $?$ \\
\hline 9.1 Dmitrij & Ivan (8.1) & 1509 & L (prison) & - & Moscou, St-Michel \\
\hline 9.2 Ivan IV & Vasilij III (8.2) & 1584 & M & T & Moscou, St-Michel \\
\hline 9.3 Jurij & Vasilij III (8.2) & 1563 & L & - & Moscou, St-Michel \\
\hline 9.4 Vladimir & Andrej (8.6) & 1569 & L (exécuté) & - & Moscou, St-Michel \\
\hline 10.1 Dmitrij & Ivan IV (9.2) & 1553 & L (min.) & - & Moscou, St-Michel \\
\hline 10.2 Ivan & Ivan IV (9.2) & 1581 & L & - & Moscou, St-Michel \\
\hline 10.3 Fedor & Ivan IV (9.2) & 1598 & L & - & Moscou, St-Michel \\
\hline 10.4 Dmitrij, st. & Ivan IV (9.2) & 1591 & L (martyr) & - & Moscou, St-Michel \\
\hline 10.5 Vasilij & Vladimir (9.4) & 1574 & L & - & Moscou, St-Michel \\
\hline
\end{tabular}

La filiation monastique dans la dynastie moscovite

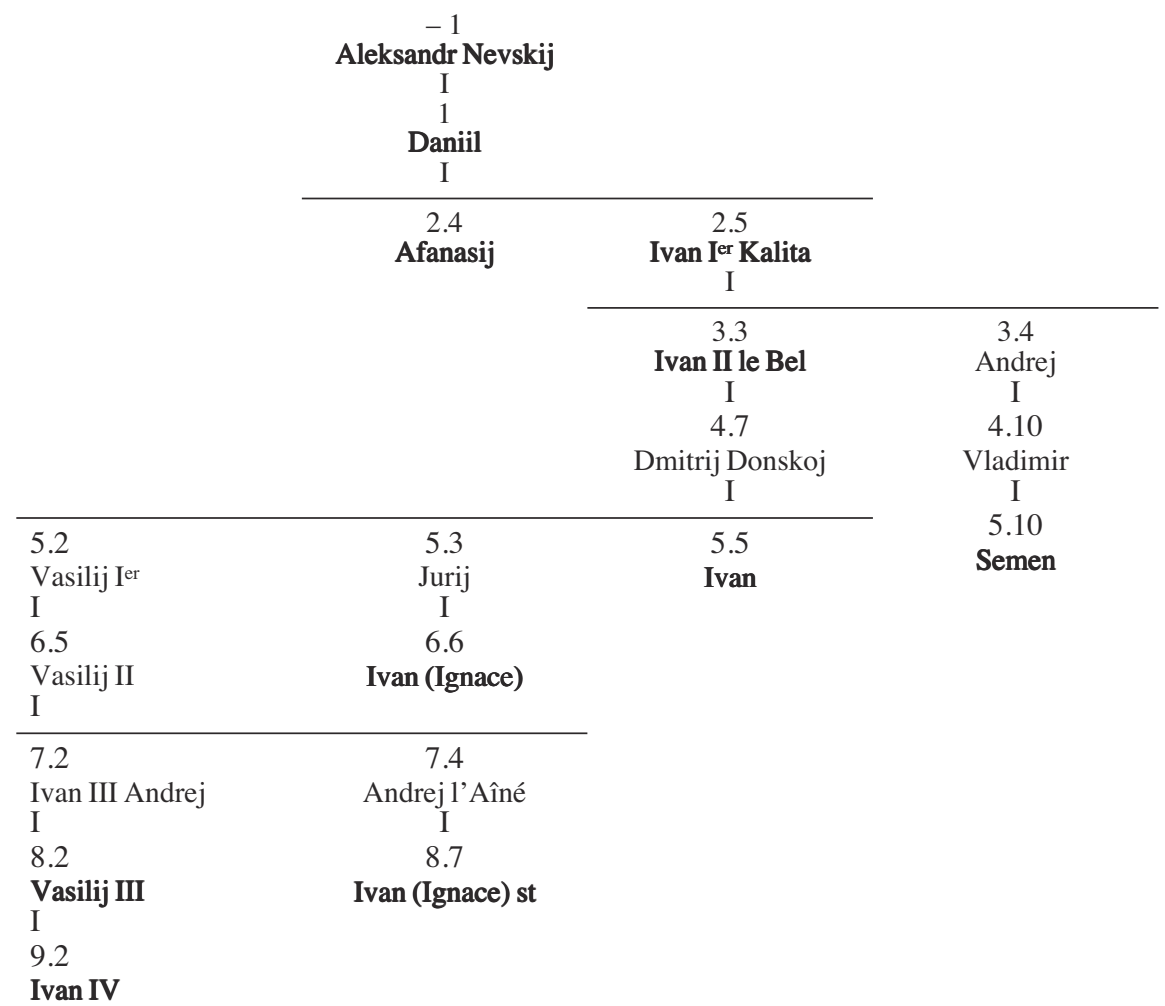

Les princes-moines apparaissent en caractères gras 